\title{
The molecular scanner in microscope mode
}

\author{
Stefan L. Luxembourg', Ali R. Vaezaddeh², Erika R. Amstalden', \\ Catherine G. Zimmermann-Ivol ${ }^{2}$, Denis F. Hochstrasser ${ }^{2,3}$ and Ron M.A. Heeren ${ }^{1 *}$ \\ ${ }^{1}$ FOM Institute for Atomic and Molecular Physics, Kruislaan 407, 1098 SJ Amsterdam, The Netherlands \\ ${ }^{2}$ Biomedical Proteomics Research Group, Department of Structure Biology \& Bioinformatics, Geneva University Medicine Faculty \& Central \\ Clinical Chemistry Laboratory, Geneva University Hospitals, Rue Michel-Servet 1, CH1211 Geneva, Switzerland \\ ${ }^{3}$ Pharmacy Section, Geneva University Sciences Faculty, CH1200 Geneva, Switzerland
}

Received 6 August 2006; Revised 14 September 2006; Accepted 18 September 2006

\begin{abstract}
The combination of microscope mode matrix-assisted laser desorption/ionization (MALDI) imaging mass spectrometry (IMS) with protein identification methodology: the molecular scanner, was explored. The molecular scanner approach provides improvement of sensitivity of detection and identification of high-mass proteins in microscope mode IMS. The methodology was tested on protein distributions obtained after separation by sodium dodecyl sulfate/polyacrylamide gel electrophoresis (SDS-PAGE). High-quality, high-spatial-resolution ion images were recorded on a TRIFT-II ion microscope after gold coating of the MALDI sample preparation on the poly(vinylidenedifluoride) capture membranes. The sensitivity of the combined method is estimated to be 5 pmol. The minimum amount of sample consumed, needed for identification, was estimated to be better than $100 \mathrm{fmol}$. Software tools were developed to analyze the spectral data and to generate broad mass range and single molecular component microscope mode ion images and single mass-to-charge ratio microprobe mode images. Copyright (C) 2006 John Wiley \& Sons, Ltd.
\end{abstract}

In matrix-assisted laser desorption/ionization (MALDI) $)^{1,2}$ imaging mass spectrometry (IMS), ${ }^{3,4}$ the two-dimensional (2-D) location and $m / z$ value of molecules present in a (biological) sample are recorded simultaneously. For complex samples, containing high-mass proteins, the recorded molecular $\mathrm{m} / \mathrm{z}$ values do not automatically result in compound identification. For this purpose additional analytical steps have to be implemented, where in general identification is pursued by the mass spectrometric (MS) analysis of molecular fragments. The fragmentation can either be introduced before (bottom-up) or during the MS analysis (top-down) (see Fig. 1). ${ }^{5}$ The latter approach can be applied in a MALDI microprobe imaging experiment on a time-of-flight/time-of-flight (ToF/ToF) or Fourier transform ion cyclotron (FTICR) mass spectrometer. However, the extended MS analysis leads to an increased sample demand and thus to lower sensitivities. In a more efficient approach the compound identification strategies indicated in Fig. 1 are applied to homogenates of the system under study. The derived knowledge on the system's protein content is then combined in sample specific atlases, which limit the number of potential candidates for identification from the $m / z$ values recorded in IMS experiments. Thereby, positive protein identification becomes more probable. The strength of this imaging/identification strategy has been shown for various samples. ${ }^{6}$

${ }^{*}$ Correspondence to: Ron M.A. Heeren, FOM Institute for Atomic and Molecular Physics, Kruislaan 407, 1098 SJ Amsterdam, The Netherlands.

E-mail: heeren@amolf.nl

Contract/grant sponsor: Nederlandse organisatie voor Wetenschappelijk Onderzoek (NWO).
One particular bottom-up compound identification strategy that has great potential for application with IMS is the so-called molecular scanner methodology. ${ }^{7,8}$ This methodology was introduced to reduce the time needed for the peptide mass fingerprinting (PMF) analysis of complex mixtures of proteins. For this purpose, proteins are first separated by sodium dodecyl sulfate/polyacrylamide gel electrophoresis (SDS-PAGE) by one- (1-DE) or twodimensional electrophoresis (2-DE). Subsequently, the proteins are digested and electrotransferred in parallel. In this so-called one-step digestion-transfer (OSDT) the entire protein content of the gel is transferred through a trypsin-containing membrane onto a poly(vinylidene difluoride) (PVDF) capture membrane by electroblotting. This results in tryptic peptide bands on the capture membrane, which can be analyzed using MALDI-MS. In contrast, in conventional in-gel digestion/PMF experiments all individual protein-containing spots have to be excised and treated with an enzymatic digest separately before MS analysis is possible. During the blotting procedure there is little migration of the peptides in the direction perpendicular to the driving electric field. As a result the protein distributions are preserved when digested and transferred to the capture membrane. Consequently, this approach is not only of interest for protein identification from homogenates, but can also be applied directly to tissue. In recent work by Stoeckli and co-workers, ${ }^{9}$ a first attempt to do so was made. In their work they describe how part of the protein content of a mouse brain tissue section was electroblotted directly,

WILEY

InterScience 


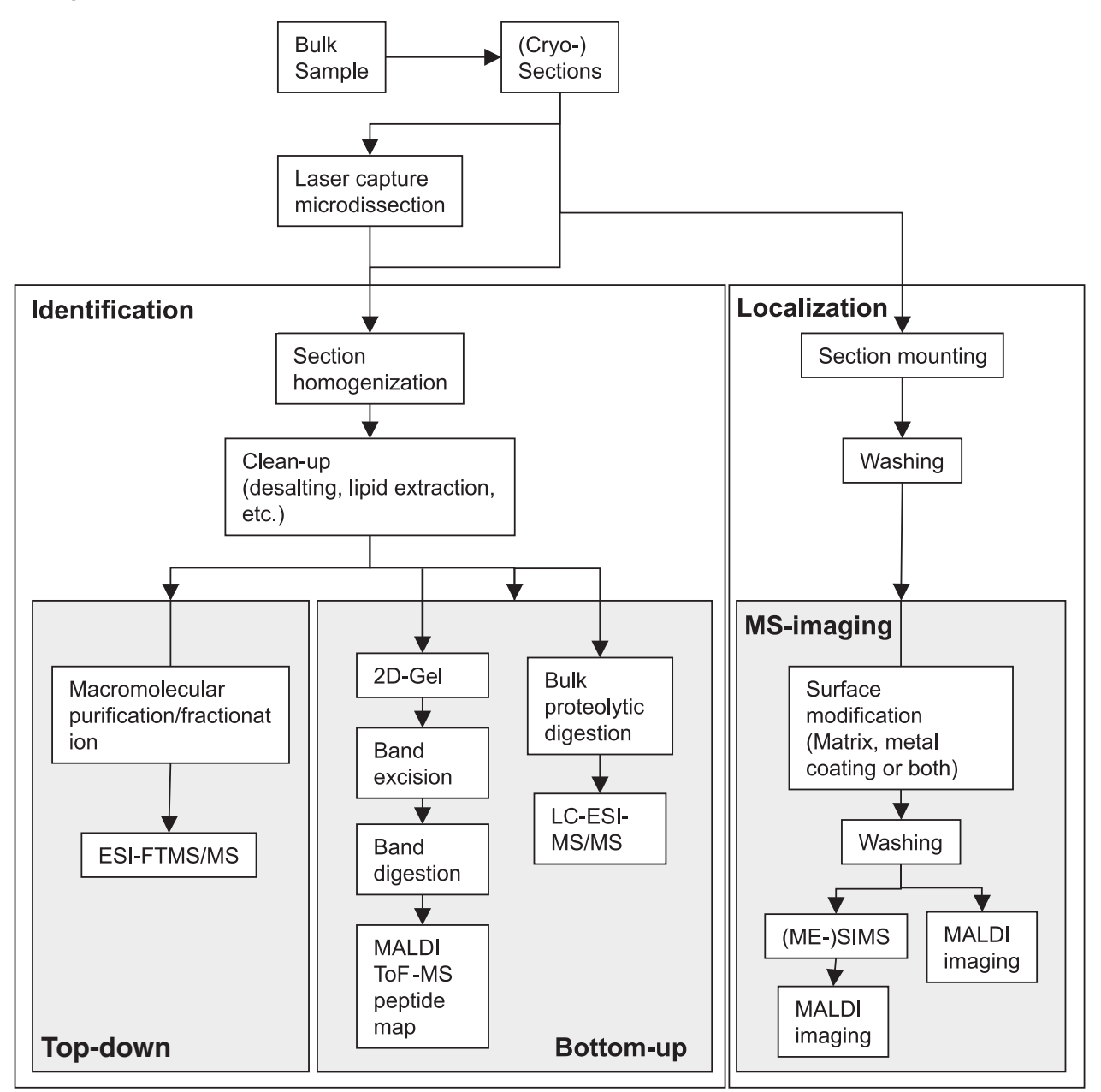

Figure 1. Different strategies for the identification and localization of proteins present in a (tissue) sample. This figure was reproduced with permission from the authors from Ref. 5 .

without a gel-separation step, through a trypsin-containing membrane onto a PVDF capture membrane. ${ }^{9}$ In the subsequent scanning microprobe MALDI imaging experiments localized peptide distributions were recorded. Imaging mass spectrometry can benefit from the molecular scanner approach in several ways. First, the presence of the tryptic fragments will help to identify the proteins originally in the sample. When combined with a sample-specific protein atlas this advantage will have its greatest effect. The spatial coordinates of the chemical information obtained, i.e. the mass spectra, further limit the complexity of the tryptic peptide mixtures that have to be considered for identification. Second, the tryptic digestion of the proteins present in the sample will increase the sensitivity; instead of single high-mass protein ions, multiple peptide ions from compounds of lower molecular mass (typically $<3000 \mathrm{Da}$ ), which can be measured with higher sensitivity, are imaged. In addition, the transblotting process separates the proteins from the other components present in the tissue, which reduces the complexity of the sample. On the other hand indications were found that some of the proteins are more easily extracted from the tissue than others. Therefore, only a part of the whole protein content of the tissue can be analyzed in this way. ${ }^{10,11}$

Copyright $(2) 2006$ John Wiley \& Sons, Ltd.
Recently, a novel approach to macromolecular IMS was demonstrated: microscope mode MALDI-MS imaging. This high-speed, high-spatial-resolution IMS technique uses position-correlated ion detection instead of positioncorrelated ion generation (conventional scanning microprobe MALDI imaging) to record mass-resolved ion images. ${ }^{4,12}$ It utilizes stigmatic ion optics to record ion images for a selected mass window at $4 \mu \mathrm{m}$ spatial resolution from within the area of the laser spot (200 $\mu \mathrm{m}$ in diameter). Larger areas are imaged by continuous movement of the sample under the UV laser spot and subsequent concatenation of the resulting ion images (scanning microscope mode imaging).

Here, we explore the combination of microscope mode MALDI-MS imaging and the molecular scanner PMF methodology. The advantages of the combined approach, mentioned above, hold especially for microscope mode imaging. The MALDI mass microscope is operated at relatively low extraction and post-acceleration potentials, which limits its sensitivity in the high- $m / z$ range. Thus, the digestion of the proteins to lower molecular mass peptides will greatly improve the sensitivity of the methodology. In addition, the high lateral resolution of the mass microscope will limit the complexity of the tryptic peptide mixtures that 
have to be considered for protein identification. The application of the molecular scanner methodology on the MALDI mass microscope makes it possible to perform both high-resolution MS imaging and protein identification in a single experimental set-up. Conversely, the high lateral resolution of the microscope mode approach can be used to gain more insight into the molecular scanner sample preparation.

This first combined molecular scanner/MALDI microscope mode imaging study is limited to protein distributions obtained from mixtures after isolation and separation on 1-DE gels. The sensitivity for the detection and identification of high-mass proteins is tested on standards. Analogous to the recent work of Scherl et al., ${ }^{13}$ we evaluated the value of gold coating the sample (PVDF membrane + matrix crystals) for mass analysis and IMS experiments on our direct extraction mass microscope. With a view towards microscope mode IMS of tissue samples prepared according to the molecular scanner protocol, we recorded high lateral resolution broad mass range as well as single compound microscope mode ion images. From the recorded datasets lower lateral resolution, single $\mathrm{m} / \mathrm{z}$ value microprobe mode data could also be extracted. The experiments described herein produce large datasets, i.e. several $\mathrm{Gb}$ of spectral information and several tens of $\mathrm{Gb}$ of image information, for an approximate sample area of $0.5 \mathrm{~cm}^{2}$. Software tools have been developed to handle, analyze and visualize these large datasets.

\section{EXPERIMENTAL}

\section{Sample preparation}

The molecular scanner sample preparation was performed by the Biomedical Proteomics Research Group at the Geneva University Hospital. The procedure is detailed elsewhere. ${ }^{7,13}$ Here, a short overview is included. The proteins present in the sample are separated using 1-DE according to Laëmmli ${ }^{14}$ with $12 \% \mathrm{~T}$ and $2.6 \% \mathrm{C}$ linear polyacrylamide gels. Subsequently, the isolated proteins are electroblotted through a trypsin-containing membrane, where they are proteolytically digested. Finally, the resulting peptides are collected on a PVDF capture membrane. A $\alpha$-cyano4-hydroxycinnamic acid (HCCA) matrix solution (10 mg/ $\mathrm{mL} \mathrm{HCCA}$ in $70 \% \mathrm{v} / \mathrm{v} \mathrm{MeOH} / 1 \% \mathrm{v} / \mathrm{v}$ trifluoroacetic acid (TFA) / $10 \mathrm{mM} \mathrm{NH}_{4} \mathrm{H}_{2} \mathrm{PO}_{4}$ ) is deposited on the membrane in regular arrays of small ( $\sim 100$ picoliter) droplets using a spotting robot, which was developed by the Geneva group. Subsequently, the samples are gold coated using a SCD 040 sputter coater (Balzers Union AG, Balzers, Liechtenstein). Proteins from a commonly used protein mixture obtained from Bio-Rad (Hercules, CA, USA) were used, to be identified from their tryptic digestion products. The protein mixture contained the following proteins: myosin from rabbit skeletal muscle (MYSS), $\beta$-galactosidase from Escherichia coli (BGAL), phosphorylase $\mathrm{b}$ from rabbit skeletal muscle (PHS2), bovine serum albumin (BSA), ovalbumin from chicken hen egg white (OVAL), carbonic anhydrase type 2 from bovine serum (CAH2), trypsin inhibitor from soybean (ITRA), lysozyme from chicken hen egg white (LYSC) and trypsin inhibitor from bovine pancreas (BPT1). It has been shown before that MYSS is usually not extracted in the electroblotting step due to its high mass and BPT1 does not separate from the migration front in 1-DE. ${ }^{15}$ The LYSC band was not analyzed in this study. Sensitivity studies were performed using a series of different amounts (200 fmol to 100 pmol) of BSA loaded on a 1-DE gel. The effect of the thickness of the gold layer was investigated on a $100 \mathrm{pmol}$ transblotted BSA sample. For this purpose the gold layer thickness was varied from 1 to $10 \mathrm{~nm}$. The protein mixtures from the esophagus adenocarcinoma cells were prepared from cells from the OACP-4c cell line. ${ }^{16}$

\section{(Imaging) mass spectrometry}

All mass spectrometric (imaging) experiments were performed on a modified TRIFT-II time-of-flight ion microscope (Physical Electronics, Chanhassen, MN, USA) equipped with a home-built UV-MALDI source for macromolecular stigmatic ion imaging. ${ }^{4}$ This mass microscope is operated with a $3 \mathrm{kV}$ ion (direct) extraction potential and an $8 \mathrm{kV}$ post-acceleration potential. The stigmatic ion optics of the TRIFT-II project $100 \times$ magnified ion optical images of the ions produced in the MALDI event onto a position-sensitive dual multichannel plate (DMCP)/phosphor screen detector. Ion images for different $\mathrm{m} / \mathrm{z}$ ions are projected onto the detector at separate times due to the differences in the flight times of the ions. The phosphor screen image is projected through a 10 times reducing lens system onto the chip of a fast CCD camera with $10 \mu \mathrm{m}$ square pixels (Imager 3, La Vision, Goettingen, Germany). In this way the overall ion and light optical magnification becomes 10 times. The flight times of the ions that constitute the ion images are obtained from the DMCP using an 8-bit PCI-digitizer card (DP 240, Acqiris, Geneva, Switzerland). One high lateral resolution microscope mode image of a selected mass window is recorded per cycle of the experiment. The mass window is set using a pair of blanker plates; a $200 \mathrm{~V}$ potential difference can be applied to deflect the ions and thereby prevent their transmission. The timing of the transmitted window is controlled using a digital delay generator (model DG535, Stanford Research Systems Inc., Sunnyvale, CA, USA). The mass window can be set from a single mass (at low $\mathrm{m} / \mathrm{z}$ ) to the whole mass spectrum. In the experiments shown here the width of the gated window was always at least $1 \mathrm{~ms}$ to prevent distortion of the ion images due to the switching of the potential of the blanker plates. This corresponds to a mass-to-charge window of approximately $20 \mathrm{~m} / \mathrm{z}$ units in the peptide mass range.

The size of the laser spot ( $\sim 200 \mu \mathrm{m}$ diameter) determines the field-of-view in a single experiment. Larger areas are imaged by continuously moving the sample stage during image acquisition. For this purpose the sample is displaced in one direction to record a series of stigmatic images, which constitute a linescan with a width of $200 \mu \mathrm{m}$ across the sample. Subsequential linescans are recorded parallel to the initial one, with sufficient overlap. A macroscopic stigmatic ion image of the sample can be constructed by stitching the individual microscope mode images together. For this purpose we developed the Spatial Image Composer (SIC) software, which will be detailed in a forthcoming publication. ${ }^{17}$ The amount of overlap of the images obtained is 
determined by the experimental settings of the repetition rate of the laser $(\sim 10 \mathrm{~Hz})$, the sample stage scan speed $(\sim 100 \mu \mathrm{m} / \mathrm{s})$ and the laser spot size. In the experiments described here, the sample is displaced approximately $10 \mu \mathrm{m}$ between subsequent laser shots. The shift between successive linescans was always set to be $100 \mu \mathrm{m}$. The software aligns the individual phosphor screen images and averages the fluorescence intensity of the overlapping parts of the images. In this way high lateral resolution ion images of large areas of the sample are obtained.

In addition to the ion microscopy data, position-resolved mass spectral data for the mass window defined in the hardware is recorded in the scanning experiments detailed above. From this dataset mass-resolved images can be constructed for each individual $\mathrm{m} / \mathrm{z}$ value within the selected mass window, at a lower lateral resolution $(\sim 200 \mu \mathrm{m})$, determined by the laser spot size. The protocol used for this closely resembles microprobe image generation and it will be referred to as such. The spectral information is combined with the sampling position for image construction. As a result of the $10 \mu \mathrm{m}$ stepsize and the $100 \mu \mathrm{m}$ displacement between linescans, regions of the sample are probed multiple times. The measured ion signal intensities for a given $\mathrm{m} / \mathrm{z}$ value are averaged in the SIC software for the overlapping laser shots. In this way rectangular image elements of approximately $10 \times 100 \mu \mathrm{m}$ are defined. The microprobe image is then constructed by the proper arrangement of these image elements

\section{RESULTS}

\section{Gold coating of the sample}

Recently, it was shown that the deposition of a thin gold layer on samples prepared according to the molecular scanner protocol dramatically improves the quality of spectral data obtained with MALDI-MS. ${ }^{13}$ The gold coating of the sample on the non-conductive PVDF membranes prevented charging effects which otherwise would result in mass shifts and strongly reduced mass resolution. For low $(8 \mathrm{kV})$ acceleration potentials the mass shift effects were found to be the largest. ${ }^{13}$ The TRIFT-II mass microscope, employed in this study, is operated at $3 \mathrm{kV}$ extraction potential and has an energy acceptance window of $160 \mathrm{eV}$. Therefore, charging effects are expected to affect the spectral quality to an even larger extent. Moreover, since the mass microscope uses electrostatic lenses for stigmatic ion imaging, residual charges on the sample will also affect the ion image quality. We have compared the spectral and image quality obtained using the mass microscope on PVDF membranes with transblotted BSA peptides without gold coating to similar samples that were coated with gold layers of different thicknesses. It was found that without gold coating charging resulted in large spot-to-spot variations in signal intensity. Often, the charging effect was large enough to completely move the kinetic energy of the ions generated out of the energy acceptance window, ${ }^{18}$ which resulted in no ions being detected. To compensate for this the sample potential had to be raised by $500-800 \mathrm{~V}$, which is indicative of a significant accumulation of negative charges on the sample. The application of a gold layer on the sample removed the need for sample potential compensation. Figure 2 shows spectra that were obtained for samples covered with 1, 2.5, 5 and $10 \mathrm{~nm}$ thick layers of gold. The application of a $1 \mathrm{~nm}$ gold layer already prevents sample charging to the extent that mass spectra can be obtained. The mass resolution obtained in these experiments did not allow a detailed study on the effect of the thickness of the gold layer on the spectral quality. A $5 \mathrm{~nm}$ gold layer was needed to prevent drifting of the ion
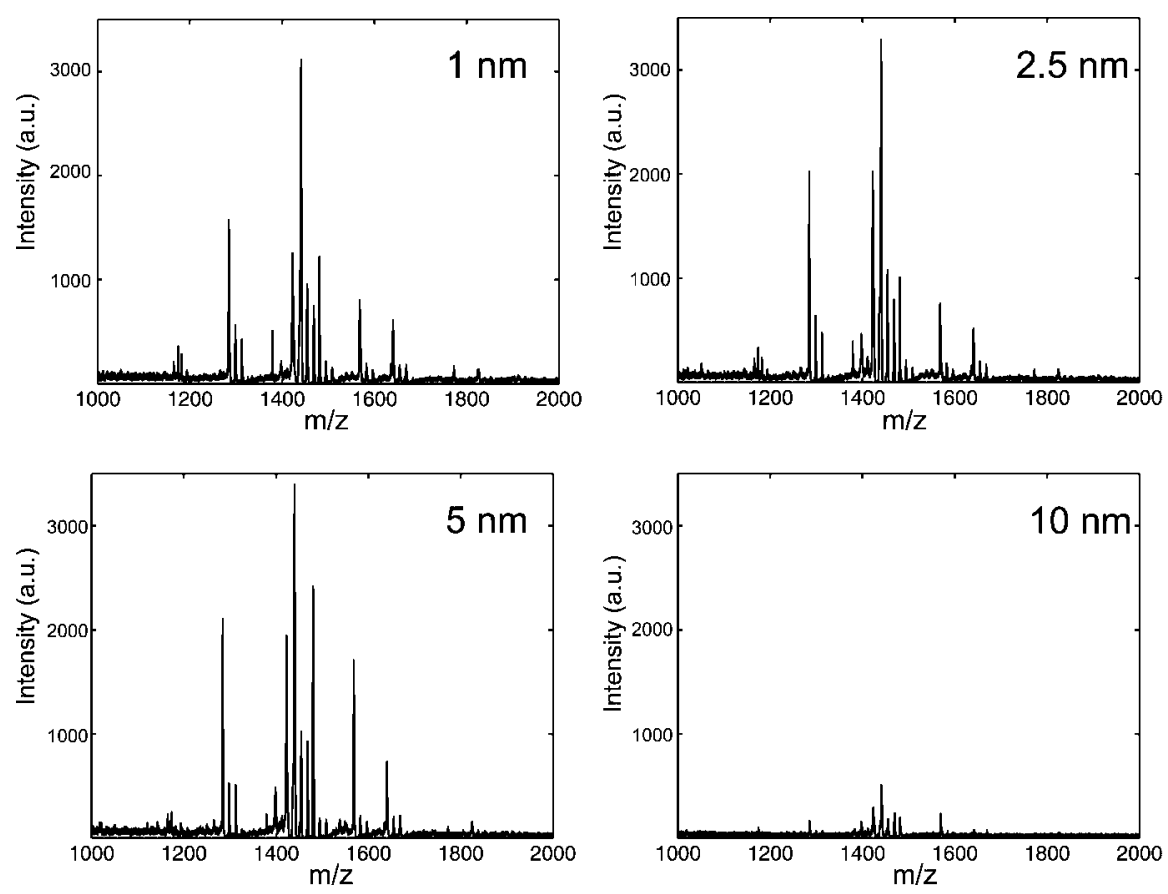

Figure 2. MALDI mass spectra of BSA digests covered with gold layers of different thicknesses. The thickness of the applied layer is indicated. The spectra results from 100 subsequent laser shots. 
Table 1. The tryptic fragments (Tf) that were detected for the different quantities of BSA loaded on the 1-DE gel. The masses and the sequences of the fragments $\mathrm{Tf} 1$ to $\mathrm{Tf} 12$ are included in Table 2. An amount of $5 \mathrm{pmol}$ was determined to be the lower limit for unambiguous identification of BSA. For an estimate of the sensitivity, see text

\begin{tabular}{|c|c|c|c|c|c|c|c|c|c|c|c|c|}
\hline $\mathrm{Q}(\mathrm{pmol})$ & Tf1 & Tf2 & Tf3 & Tf4 & Tf5 & Tf6 & Tf7 & Tf8 & Tf9 & Tf10 & Tf11 & Tf12 \\
\hline 100 & + & + & + & + & + & & + & + & + & + & + & + \\
\hline 50 & + & + & + & + & + & + & + & + & + & + & & \\
\hline 12.5 & + & & & & + & & + & + & + & + & + & + \\
\hline 6.25 & + & + & & & + & & + & + & + & + & + & \\
\hline 5 & + & & & & + & & + & + & + & + & & \\
\hline 0.5 & + & & & & & & & + & + & + & & \\
\hline
\end{tabular}

images completely. The high quality of the ion images obtained with a $5 \mathrm{~nm}$ gold layer will be demonstrated later in this paper. The application of a $10 \mathrm{~nm}$ thick gold layer significantly reduced the ion yield.

\section{Sensitivity}

In order to estimate the sensitivity of the molecular scanner/ microscope mode approach different amounts of BSA were loaded on a gel. After OSDT, matrix and gold deposition peptide spectra of the BSA digests were obtained. For this purpose the sample was moved under the laser spot. One linescan consisted of 500-1250 laser shots. Table 1 lists the peptides observed for the different amounts of BSA loaded. The number of BSA peptides present in the spectra varied from 4 to 11. In Table 2, the masses and the amino acid sequences of the detected tryptic fragments, as derived from the SwissProt database, are included. When $200 \mathrm{fmol}$ of BSA was loaded no peptides were detected. For all samples the strongest signal was obtained for the $m / z 1480$ peptide. For the larger amounts of BSA ( $\geq 5$ pmol) a mass accuracy of better than 100 ppm and mass resolution of 2000 were achieved. For lower amounts of sample the laser intensity had to be increased to record sufficient peptide signal. These elevated laser intensities resulted in deterioration of the mass accuracy and mass resolution. We evaluated if the recorded peptide ion signals, for different amounts of BSA loaded, were sufficient for positive identification in a Mascot PMF

Table 2. The masses and amino acid sequences of the tryptic fragments of BSA which were detected in the sensitivity tests

\begin{tabular}{lcl}
\hline & Mass $(\mathrm{Da})$ & Sequence \\
\hline Tf1 & 927 & YLYEIAR \\
Tf2 & 1050 & EACFAVEGPK \\
Tf3 & 1194 & DTHKSEIAHR \\
Tf4 & 1250 & FKDLGEEHFK \\
Tf5 & 1284 & HPEYAVSVLLR \\
Tf6 & 1306 & HLVDEPQNLIK \\
Tf7 & 1440 & RHPEYAVSVLLR \\
Tf8 & 1480 & LGEYGFQNALIVR \\
Tf9 & 1568 & DAFLGSFLYEYSR \\
Tf10 & 1640 & KVPQVSTPTLVEVSR \\
Tf11 & 1824 & RPCFSALTPDETYVPK \\
Tf12 & 1900 & LGEYGFQNALIVRYTR \\
\hline
\end{tabular}

Copyright (C) 2006 John Wiley \& Sons, Ltd.
Search $^{19}$ using the SwissProt database. For an amount of 5 pmol of BSA the protein could still be identified unambiguously; for lower amounts the number of peptides detected in combination with the reduced mass accuracy did not yield positive identification. Therefore, the sensitivity of the combined methodology was estimated to be 5 pmol. Based on the following considerations, we estimated the amount of sample that was consumed in order to come to a positive identification. The band on the PVDF membrane containing the BSA peptides extended over approximately $4 \mathrm{~mm}$ in the vertical direction. Most intense signals were obtained from the middle $1.5 \mathrm{~mm}$. In the horizontal direction the band extended approximately $8 \mathrm{~mm}$. For the analysis of the $5 \mathrm{pmol}$ sample 500 laser shots were taken, while the sample was moved at $50 \mu \mathrm{m} / \mathrm{s}$. In one linescan approximately $0.7 \mathrm{~mm}^{2}$ of the sample was analyzed. For the lower limit for BSA identification the analyzed area contained an amount of peptide material corresponding to $100 \mathrm{fmol}$ of BSA on the original gel. In this estimate it is assumed that all the protein material is transferred from the gel to the membrane. Since this is not the case (see below) the $100 \mathrm{fmol}$ is a conservative estimate. Moreover, analysis of the 5 pmol dataset revealed that selected mass spectra demonstrated a sufficient signal-to-noise ratio to detect all six peptides on a single shot basis. Thus our estimate is that the amount of material used in a single shot experiment is 200 atmol. The transfer efficiency of the proteins from the gel to the capture membrane has been studied using fluorescently labeled proteins. ${ }^{20}$ It was estimated that $5 \%$ of the proteins had remained in the gel after the transfer. In a very recent study an overall efficiency of $75 \%$ for the transfer from the gel to the capture membrane was found. It was found that a small percentage of the tryptic peptides pass through the pores of the capture membrane and are lost for further analysis. This also indicates that the peptides are not concentrated in the topmost part of the capture membrane but will be distributed across its $100 \mu \mathrm{m}$ thickness. Efforts to further improve the sensitivity of the methodology should therefore be directed towards quality improvement of the capture membrane.

\section{Protein identification from a mixture}

A commercial mixture of proteins was treated according to the sample preparation protocol presented above. For each of the proteins approximately 100 pmol was loaded on the gel in a pre-mixed solution; six bands were analyzed using our 


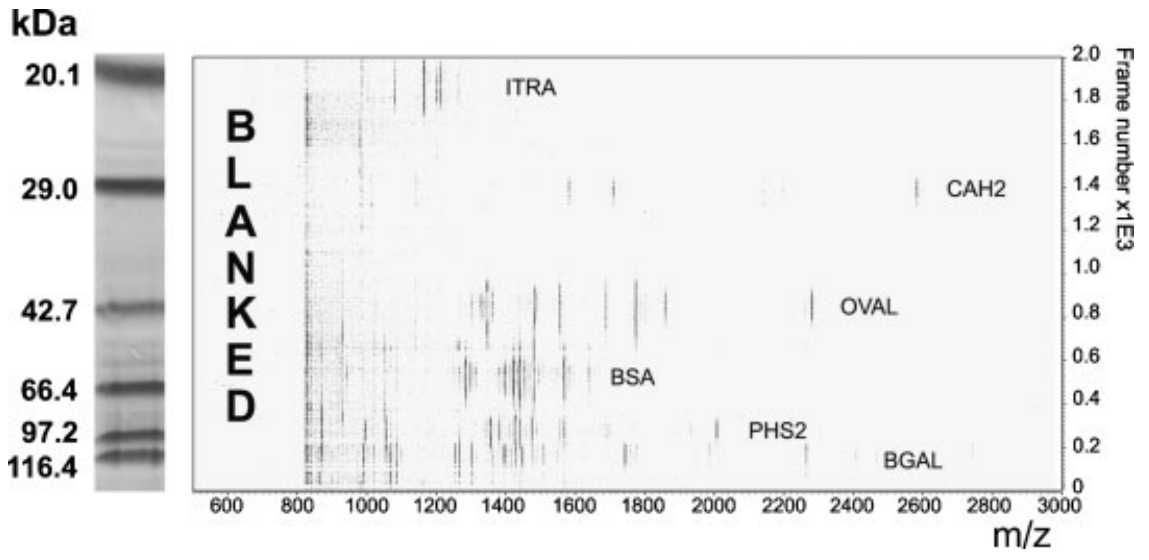

Figure 3. The proteins present in a commercial mixture were separated on a 1-DE gel and subsequently analyzed using the molecular scanner methodology. On the left side an image of the gel stained with Coomassie Blue is shown. The positions of the peptide bands detected on the mass microscope and visualized using Awe3D software agree with the positions on the gel. The peptides localized in bands facilitated the identification of the proteins indicated.

MALDI mass microscopy set-up. The left side of Fig. 3 displays the result of the 1-DE gel separation, in which the six targeted bands are visible and labelled. The sample was continuously moved under the laser spot at a speed of $100 \mu \mathrm{m} / \mathrm{s}$, which yielded 2000 mass spectra for a single microscope linescan. Ions with $\mathrm{m} / \mathrm{z}$ values below $\mathrm{m} / \mathrm{z} 800$ were filtered from the secondary ion beam using a pair of blanker plates (see Experimental section) to prevent saturation of the detector and, for this reason, do not appear in the spectrum. For the spectral analysis and representation of the acquired data we used specially designed software: Awe3D, ${ }^{21}$ developed in our lab for the analysis of 2-D liquid chromatography FTICR-MS datasets and adapted for visualization of the data acquired in a molecular scanner experiment. The right hand side of Fig. 3 shows a virtual gel representation of the data in Awe3D. In this representation of the data the occurrence of specific $m / z$ values in the spectrum (horizontal axis) can be easily linked to specific laser shot numbers. The six peptide-containing bands are clearly visible, and their positions correspond to those on the gel. The software can be used to zoom in on individual peptide bands and inspect single laser shot mass spectra, which can be used to accurately determine the mass of the peptides recorded. The peptides detected yielded sufficient sequence coverage for a positive identification of the proteins in all six bands in Fig. 3.

\section{Microprobe and microscope mode IMS}

As was detailed in the Experimental section the mass microscope is capable of different imaging MS modes. The phosphor screen/CCD camera configuration is used to capture high-resolution stigmatic/microscope mode ion images; blanker plates are used to restrict the detection of ions to a certain $\mathrm{m} / \mathrm{z}$ window: ranging from single mass to full mass range $(<m / z \quad 100$ 000). Lower spatial resolution microprobe mode images for $m / z$ values within the selected mass window can be constructed from the mass spectra, which are recorded simultaneously with the images. The two different imaging concepts are demonstrated in Fig. 4.
Proteins extracted from human esophagus adenocarcinoma cells were loaded on a gel for separation and subsequent OSDT onto a PVDF membrane. After matrix solution and a $5 \mathrm{~nm}$ gold layer had been applied to the sample, the $35 \mathrm{~mm}$ long capture membrane was scanned at $100 \mu \mathrm{m} / \mathrm{s}$ in one direction. A linescan consisting of 3300 individual images and spectra was obtained. The distance between subsequent single laser shot images in this dataset was $11.1 \mu \mathrm{m}$. In total 10 linescans were recorded, yielding 33000 individual images and spectra. Each linescan was recorded in under $5 \mathrm{~min}$, which resulted in a total experiment time of approximately $50 \mathrm{~min}$. The total dataset recorded consisted of $20 \mathrm{~Gb}$ of image data and $3 \mathrm{~Gb}$ of spectral data. For the handling and representation of these huge datasets we used the SIC software. Using this software large area microscope mode ion images for the hardware-selected mass window as well as microprobe mode images for all individual $\mathrm{m} / \mathrm{z}$ values were produced. The result for the esophagus sample is shown in Fig. 4(A) (first lane). Figure 4(B) includes a zoom of a $0.5 \times 1 \mathrm{~mm}$ area of this result. It clearly demonstrates that the high spatial resolution of the individual ion images is maintained. It reveals a ring-like structure, which results from the matrix deposition procedure. The diameter of the rings is close to $100 \mu \mathrm{m}$. It is immediately clear that resolvation and redistribution of the analytes within these structures will limit the spatial information that is obtained with this matrix deposition method. Microprobe images for selected $\mathrm{m} / \mathrm{z}$ values are constructed from $11.1 \times 100 \mu \mathrm{m}$ image elements, which are defined by the scanning procedure. Lanes 2 to 5 of Fig. 4(A) show microprobe mode images of the sample for the peptide masses indicated. With the approximately 50 times lower spatial resolution of the microprobe mode analysis the sample preparation artifacts (ring structures) are not resolved. Although many different peptides were detected for the esophagus sample this did not result in identification of their protein precursors. High salt concentrations in the sample prevented the formation of distinguishable protein bands during the 1-DE gel separation step. 

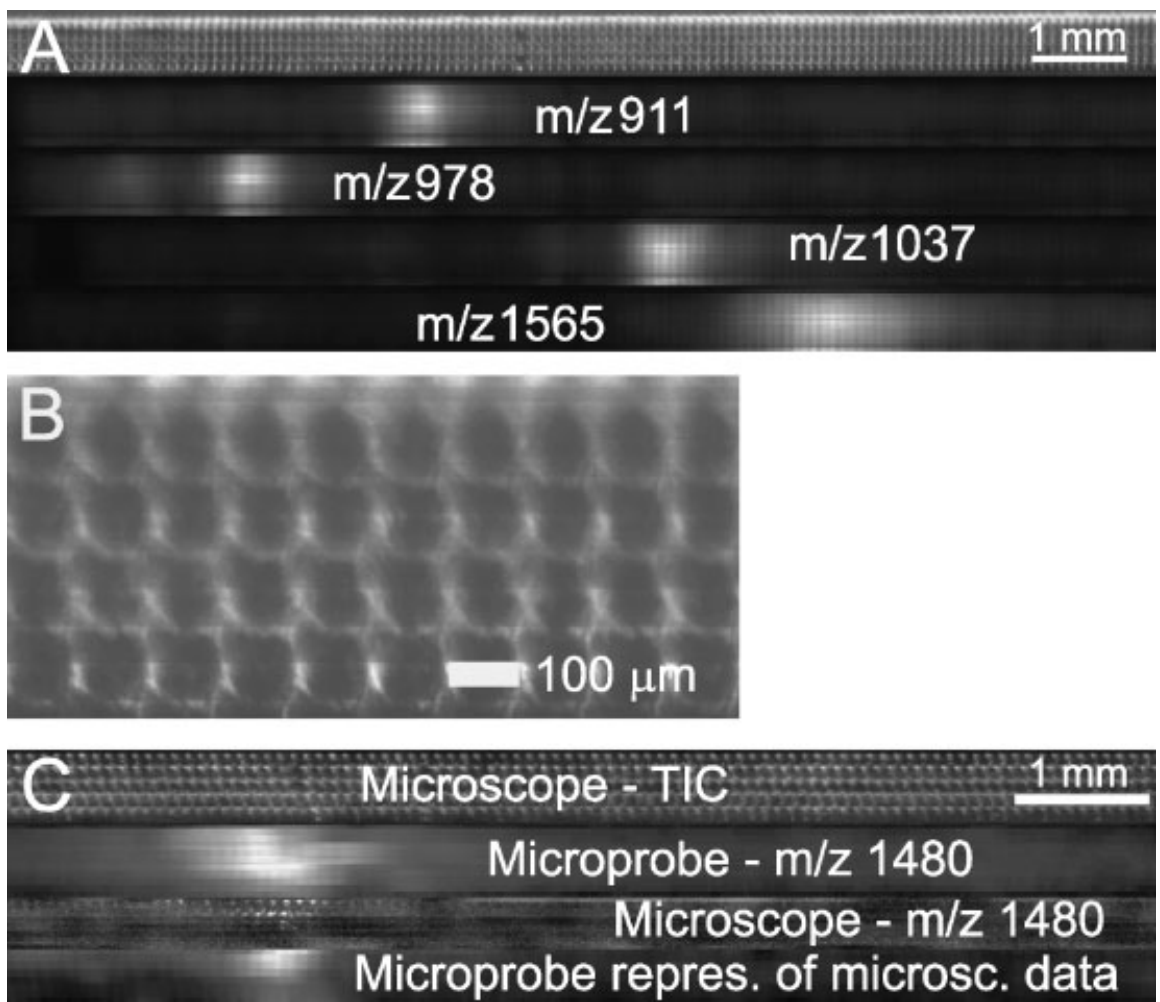

Figure 4. (A) Molecular scanner esophagus sample. The top lane contains the microscope mode TIC image. The four subsequent lanes contain single $\mathrm{m} / \mathrm{z}$ microprobe mode images. (B) A zoom of a $0.5 \times 1 \mathrm{~mm}$ area of the microscope mode TIC image shows the $100 \mu \mathrm{m}$ diameter matrix crystal rings. (C) TIC microscope mode, microprobe mode $\mathrm{m} / \mathrm{z} 1480$ and microscope mode $\mathrm{m} / \mathrm{z} 1480$ images obtained on a commercial protein mixture sample. The bottom lane contains a microprobe representation of the microscope mode $\mathrm{m} / \mathrm{z} 1480$ data (see text for details).

The mass window for microscope mode ion imaging can be set such that the distribution of a single compound can be imaged at high lateral resolution. This application is particularly advantageous when it is preceded by a microprobe analysis in which interesting molecular distributions have been first localized. For the single component selection the ion blankers are unblanked only for a very short period in time: $1 \mu \mathrm{s}$. Figure 4(C) contains images that result from nine linescans of 3500 individual images and spectra obtained on the broad range protein standards sample described earlier in this study. The first lane contains the TIC (total ion count) image $(m / z>800)$. The ring structure resulting from the matrix deposition procedure is again clearly visible. The second image is a microprobe representation of the data, showing the distribution of a specific peptide at $m / z 1480$. This peptide results from the tryptic digestion of BSA and can be used to localize the BSA band. The image in the third lane of Fig. 4(C) is a microscope mode image of the distribution of the peptide found at $m / z 1480$. This image was recorded in a separate measurement of six linescans at a fresh position on the sample. Therefore, its intensity and vertical distribution cannot be compared directly with those of the first two images. The last lane contains the microprobe mode representation of the microscope mode single component $\mathrm{m} / \mathrm{z} 1480$ dataset. From this image it is apparent that the locations of the band in the two different imaging modes are in agreement. In the single component microscope mode

Copyright (C) 2006 John Wiley \& Sons, Ltd. image the details of the sample preparation are visible. These results are a good example of the complementarity of the microprobe and microscope operation; trading mass range for high resolving power.

\section{DISCUSSION AND CONCLUSIONS}

Using the molecular scanner PMF methodology high molecular weight proteins like $\beta$-galactosidase (116 kDa) are identified on a TRIFT-II ion microscope, which is operated at low extraction field and limited postacceleration. The multiple peptide digestion products per protein in combination with the reduced masses of the analyte species studied facilitate this. The sensitivity for unambiguous identification of BSA is estimated to be 5 pmol. The actual amount of sample consumed in the MALDI analysis resulting in positive identification was almost two orders of magnitude lower.

The combined molecular scanner/microscope mode IMS methodology offers a means to record the distributions of high molecular weight proteins with high spatial detail. For this purpose the molecular scanner sample preparation has to be extended with the application of a $5 \mathrm{~nm}$ gold layer.

High spatial resolution (microscope mode) ion images were recorded for mass ranges that were set in the instrument hardware. Microprobe mode images for all $\mathrm{m} / \mathrm{z}$ values within the set mass range were constructed from the 
spectral data that was recorded simultaneously. This latter mode of operation is particularly useful for a first, lowspatial-resolution analysis of the sample in which interesting distributions of masses can be distinguished. In a subsequent experiment an interesting species can be selected for high-resolution imaging in microscope mode. The TIC and single species microscope mode images display rings of matrix crystals with a diameter of approximately $100 \mu \mathrm{m}$. These results demonstrate that the current matrix deposition method is not suited for high lateral resolution IMS. To take advantage of the high lateral resolution that can be achieved in microscope mode imaging, the sample preparation will have to be optimized. Electrospray and sonic spray matrix deposition methods produce micron-size matrix crystals from a spray of very small droplets. These sample preparation methods are compatible with high lateral resolution imaging, ${ }^{22}$ although the limited sample wetting can result in a loss of sensitivity due to reduced incorporation of the proteins/peptides present in the sample. ${ }^{23}$ The sensitivity that can be obtained using electrospray and sonic spray matrix deposition will have to be evaluated. In this respect the optimization of the capture membrane is of great importance. Developments should be aimed at preventing the transferred peptides from passing through the membrane and concentration near the membrane surface.

The combination of the molecular scanner and MALDI microscope mode imaging methodology extends high spatial resolution MS imaging to high molecular weight proteins. Its potential for application directly on biological tissue is clearly recognized and will be the subject of future studies.

\section{Acknowledgements}

The authors kindly acknowledge W. N. Dinjens for his gift of the OACP-4c cells. This work is part of research program nr. 49 "Mass spectrometric imaging and structural analysis of biomacromolecules" of the Stichting voor Fundamenteel Onderzoek der Materie (FOM), which is financially supported by the Nederlandse organisatie voor Wetenschappelijk Onderzoek (NWO).

\section{REFERENCES}

1. Karas M, Bachmann D, Hillenkamp F. Anal. Chem. 1985; 57: 2935.

2. Karas M, Bachmann D, Bahr U, Hillenkamp F. Int. J. Mass Spectrom. Ion Processes 1987; 78: 53.

3. Caprioli RM, Farmer TB, Gile J. Anal. Chem. 1997; 69: 4751.

4. Luxembourg SL, Mize TH, McDonnell LA, Heeren RMA. Anal. Chem. 2004; 76: 5339

5. Heeren RMA, McDonnell LA, Amstalden E, Luxembourg SL, Altelaar AFM, Piersma SR. Appl. Surf. Sci. 2006; in press.

6. Chaurand P, Caprioli RM. Electrophoresis 2002; 23: 3125.

7. Binz P-A, Müller M, Walther D, Bienvenut WV, Gras $R$, Hoogland C, Bouchet G, Gasteiger E, Fabbretti R, Gay S, Palagi P, Wilkins MR, Rouge V, Tonella L, Paesano S, Rossellat G, Karmime A, Bairoch A, Sanchez J-C, Appel RD, Hochstrasser DF. Anal. Chem. 1999; 71: 4981.

8. Bienvenut WV, Sanchez J-C, Karmime A, Rouge V, Rose K, Binz P-A, Hochstrasser DF. Anal. Chem. 1999; 71: 4800.

9. Rohner TC, Staab D, Stoeckli M. Mech. Ageing Dev. 2005; 126: 177.

10. Chaurand P, Fouchecourt S, DaGue BB, Xu BJ, Reyzer MJ, Orgebin-Crist M-C, Caprioli RM. Proteomics 2003; 3: 2221.

11. Chaurand P, DaGue BB, Pearsall RS, Treadgill DW, Caprioli RM. Proteomics 2001; 1: 1320.

12. Luxembourg SL, McDonnell LA, Mize TH, Heeren RMA. J. Proteomics Res. 2005; 4: 671.

13. Scherl A, Zimmermann-Ivol CG, Dio JD, Vaezzadeh AR, Binz P-A, Amez-Droz M, Cochard R, Sanchez J-C, Glückmann M, Hochstrasser DF. Rapid. Commun. Mass Spectrom. 2005; 19: 605

14. Laëmmli UK. Nature 1970; 277: 680.

15. Bienvenut WV, Déon C, Sanchez J-C, Hochstrasser DF. Anal. Biochem. 2002; 307: 297.

16. de Both NJ, Wijnhoven BPL, Sleddens H, Tilanus HW, Dinjens WNM. Virchows Archiv-an International Journal of Pathology 2001; 438: 451.

17. Klinkert I, Luxembourg SL, Altelaar AFM, Amstalden ER, Piersma SR, Konijnenburg M, McDonnell LA, Heeren RMA. Rev. Sci. Instrum. 2006/2007; submitted.

18. Luxembourg SL, Heeren RMA. Int. J. Mass Spectrom. 2006; 253: 181.

19. Available: http://www.matrixscience.com.

20. Nadler TK, Wagenfeld BG, Huang Y, Lotti RJ, Parker KC, Vella GJ. Anal. Biochem. 2004; 332: 337.

21. Mize TH, Taban I, Duursma M, Seynen M, Konijnenburg M, Doornik CV, Rooij Gv, Heeren RMA. Int. J. Mass Spectrom. 2004; 235: 243.

22. Altelaar AFM, van Minnen J, Jimenez CR, Heeren RMA, Piersma SR. Anal. Chem. 2005; 77: 735.

23. Schwartz SA, Reyzer ML, Caprioli RM. J. Mass Spectrom. 2003; 38: 699. 